Ganglioneuroblastoma may infiltrate locally as well as metastasize by way of haematogenous and lymphatic channels, as does neuroblastoma. Ganglioneuroblastomas are slow growing tumours and long-term postoperative survivals have been reported in many clinical series. ${ }^{2,3,8}$ The prognosis is generally favourable with 2 year survival of $92 \%$ and 5 year survival of $88 \%$ in a large series. ${ }^{1}$ Spontaneous regression of neuroblastoma is well documented and there is evidence in the literature that many of the so-called cures in neuroblastoma are in fact spontaneous regression. In our patient no mature ganglioneuroma was found on excision and response occurred only after chemotherapy, too close a coincidence for spontaneous regression.

\section{References}

1. Adam, A. \& Hochholzer, L. Ganglioneuroblastoma of the posterior mediastinum. A clinicopathological review of 80 cases. Cancer 1981, 47: 373-381.

2. Johannson, J.H., Rekate, H.L. \& Roessmann, U. Gangliomas, pathological and clinical correlations. J. Neurosurg 1981, 54: $58-63$.

3. Kalyan-Raman, U.P. \& Olivero, W.C. Ganglioglioma: a correlative clinicopathological and radiological study of ten surgically treated cases with follow-up. Neurosurgery 1987, 20: $428-433$.

4. Kilton, L.J., Aschenbrener, C. \& Burns, C.P. Ganglioneuroblastoma in adults. Cancer 1976, 37: 974-983.
Literature regarding the role of chemotherapy in ganglioneuroblastoma is limited and results are $\mathbb{1}$ conflicting. The excellent response obtained in the ${ }_{c}$ present case warrants a closer look at chemotherapy as the first line of treatment. We used a fairly aggressive and broad-spectrum protocol ando it is not clear which drug was the most active. The음 change in drugs after the first three courses of a $\frac{\bar{\rho}}{C}$ different protocol were preplanned to reduce the ${ }_{0}^{\mathbb{D}}$ toxicity and to maximize the response. In view of the fact that the tumour is so rare we may not have $\rightarrow$ an answer to these questions for a long time to? come. Our experience is particularly relevant to a $\overrightarrow{\vec{\omega}}$ situation where the tumour is unresectable or $\stackrel{\omega}{\sigma}$ surgery is hazardous.

5. Kissane, J. \& Smith, M. Pathology of infancy and childhood. $\vec{G}$ C.V. Mosby, St Louis, 1967, pp. 706-713.

6. Ng, T.H.K., Fung, C.F., Goh, W. \& Wong, V.C.N. Gang- $\underset{\infty}{+}$ lioneuroma of spinal cord. Surg Neurol 1991, 35: 147-151.

7. Russell, D.S. \& Rubinstein, L.J. Peripheral tumours of the neurone series. In: Pathology of Tumours of the Nervous System. Williams and Wilkins, Baltimore, 1971, pp.305-333. O

8. Sutton, L.N., Packer, R.J. Rorke, L.B., Bruce, D.A. \& Schut, $\frac{\mathbb{D}}{0}$ I. Cerebral gangliogliomas during childhood. Neurosurgery $\vec{\Phi}$ 1983, 13: $124-128$.

\title{
Is clubbing a feature of the anti-phospholipid antibody syndrome?
}

\author{
A.W. Harris, T.A.C. Harding, M.D. Gaitonde and J.D. Maxwell
}

Department of Medicine, St George's Hospital, London SW17 0QT, UK

\begin{abstract}
Summary: A patient with the anti-phospholipid antibody syndrome and digital clubbing is described. No recognized cause for the clubbing was found. It is suggested that platelet aggregation and microthrombi formation as a result of anti-phospholipid antibody may be involved in the pathogenesis of the digital clubbing. This may be a new feature of the anti-phospholipid antibody.
\end{abstract}

\section{Introduction}

Digital clubbing is a recognized feature of many respiratory, cardiac and gastrointestinal diseases. We describe a case of clubbing in a man with the anti-phospholipid antibody syndrome, with no

Correspondence: A. Harris, B.Sc., M.R.C.P., Ogle Office, Knightsbridge Wing, St George's Hospital, Tooting, London SW17 0QT, UK.

Accepted: 20 January 1993 other recognized cause for his clubbing, and sug- N gest that this may be a feature of this condition.

\section{Case report}

A 48 year old retired safe-maker was referred for investigation of abnormal liver function tests. $\operatorname{In}_{\Omega}^{\mathbb{D}}$ 
1984, aged 40 , he had a left middle cerebral artery embolic stroke, confirmed by computed tomographic scan. The following year he was diagnosed as having temporal lobe epilepsy and was started on carbamazepine. Four years later, he suffered from two transient ischaemic attacks. No further investigation was undertaken at that time. In 1989 he was seen by a dermatologist for recurrent bilateral, lower leg ulceration. There was no history of hypertension, diabetes mellitus, hyperlipidaemia, ischaemic heart disease or deep vein thrombosis.

General examination revealed finger and toe clubbing first noticed about 6 months previously (Figure 1). There were signs of mitral and tricuspid incompetence, a mild expressive dysphasia, spastic left hemiparesis and bilateral lower leg venous ulceration.

Investigations revealed normal haematology other than thrombocytopenia $\left(84 \times 10^{9} / 1\right)$ and prolonged activated partial thromboplastic time (APTT) to 97 seconds (normal: $34-48$ ), with the presence of a lupus anticoagulant. The alkaline phosphatase and gamma-glutamyl transferase were elevated, $264 \mathrm{IU} / 1$ (normal 30-120) and $540 \mathrm{IU} / 1$ (normal <30) respectively. The remaining liver function tests were consistently normal. Hepatitis B, C and VDRL were negative. Arterial blood gases and electrocardiogram were normal. Chest radiograph showed an enlarged heart with upper lobe blood diversion. Radiographs of the wrists were normal. Abdominal ultrasound confirmed pulsatile hepatomegaly and dilated hepatic veins, but no thrombosis. Subsequent venography of the deep leg veins and inferior vena cava was normal. Echocardiography confirmed the clinical

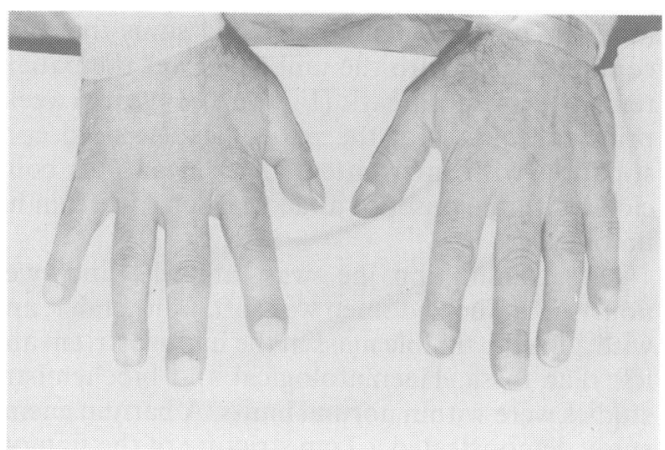

Figure 1 Digital clubbing in a patient with the antiphospholipid antibody syndrome.

\section{References}

1. Hughes, G.R.V. Thrombosis, abortion, cerebral disease and the lupus anticoagulant. $\mathrm{Br}$ Med J 1983, 287: 1088-1089.

2. Hughes, G.R.V., Harris, E.N. \& Gharavi, A.E. The anticardiolipin syndrome. J Rheumatol 1986, 13: 486-489. findings, and demonstrated no valvular vegetations. Rigid sigmoidoscopy and biopsy were normal. A liver biopsy showed features of chronic venous outflow block, and enzyme induction. Immunology revealed negative ANA, ds-DNA and ANCA but strongly positive anti-cardiolipin IgG antibody titres (79 units; normal <10).

\section{Discussion}

Recurrent venous and arterial thromboses, thrombocytopenia, heart valve abnormalities, epilepsy and skin ulceration are all well recognized features of the anti-cardiolipin syndrome. ${ }^{1-4}$ The patient we have described did not suffer from systemic lupus erythematosus or take any medication associated with anti-phospholipid antibodies. ${ }^{5}$ Therefore a diagnosis of the primary anti-phospholipid antibody syndrome was made.

Binding of the anti-cardiolipin antibody to platelet phospholipid may damage platelets and increase their adhesiveness leading to aggregation and microthrombi formation. ${ }^{6-8}$ Thrombocytopenia is a common finding in patients with anticardiolipin antibodies and thrombosis. ${ }^{9}$ Dickinson and Martin ${ }^{10}$ proposed that the release of plateletderived growth factor (PDGF) from impacted platelet clumps in the fingertips leads to increased capillary permeability and connective tissue hypertrophy which may result in clubbing. Support for this hypothesis was given by Fox et al. ${ }^{11}$ They studied autopsy samples from patients with unequivocal digital clubbing and randomly selected controls. Numerous platelet microthrombi were demonstrated in all the clubbed specimens, and in none of the controls. They concluded that finger clubbing is associated with platelet aggregation in the capillary network of the nail bed, with possible release of PDGF.

Our patient was not congenitally clubbed. There was no evidence of any chronic respiratory condition or bronchial carcinoma, inflammatory bowel disease or malabsorption, cirrhosis, infective endocarditis or congenital cardiac anomalies. In the absence of these conditions, we suggest that the finger and toe nail clubbing may be a new feature of the anti-phospholipid antibody syndrome.

\section{Acknowledgements}

The authors thank Professor C. Seymour for her help with the case.

3. Alegre, V.A., Gastineau, D.A. \& Winklemann, R.K. Skin lesions associated with circulating lupus anticoagulant. $\mathrm{Br} J$ Dermatol 1989, 120: 419-429. 
4. Galve, E., Ordi, J., Barquinero, J., Evangelista, A., Vilardell, M. \& Soler-Soler, J. Valvular heart disease in the primary antiphospholipid syndrome. Ann Intern Med 1992, 116: 293-298.

5. Canoso, R.T. \& Sise, H.S. Chlorpromazine induced lupus anticoagulant and associated immunological abnormalities. Am J Hematol 1982, 13: 121-129.

6. Anon. Lupus anticoagulant. Lancet 1984, 389: 1157-1158.

7. Elias, M. \& Eldor, A. Thromboembolism in patients with the 'lupus'-type circulating anticoagulant. Arch Intern Med 1984, 144: 510-515.

8. Weiner, H.N., Vardinon, N. \& Yust, I. Platelet antibody binding and spontaneous aggregation in 21 lupus anticoagulant patients. Vox Sang 1991, 61: 111-121.
9. Harris, E.N., Asherson, R.A., Gharavi, A.E., Morgan, S.H. Derve, G. \& Hughes, G.R.V. Thrombocytopenia in SLE andd related autoimmune disorders: association with anticardiolipin antibodies. Br J Haematol 1985, 59: 227-230.

10. Dickinson, C.J. \& Martin, J.F. Megakaryocytes and platelet: clumps as the cause of finger clubbing. Lancet 1987, 573: 1434-1435.

11. Fox, S.B., Day, C.A. \& Gatter, K.C. Association between platelet microthrombi and finger clubbing. Lancet 1991, 336 $\overline{\frac{\pi}{5}}$ 313-314.

\title{
The development of spontaneous colo-umbilical fistula
}

\author{
John B. Pracyk, Stephen G. Pollard and Sir Roy Y. Calne
}

Department of Surgery, University of Cambridge, Level 9, Addenbrooke's Hospital, Hills Road, Cambridges $C B 22 Q Q, U K$

\begin{abstract}
Summary: A patient with colo-umbilical fistula is reported. This presentation is unique because io documents the development of a fistula from a colonic diverticulum. Sigmoid colectomy was undertakei successfully.
\end{abstract}

\section{Introduction}

Entero-umbilical fistulas are rare $^{1}$ and in most cases there is a predisposing cause. Here we describe a case of a fistula that developed from a diverticulum of the sigmoid colon that discharged through the umbilicus. To our knowledge, a spontaneous colo-umbilical fistula has not been described previously. We discuss the pathophysiology and the rationale for surgical management.

\section{Case report}

A 76 year old male retired engineer was admitted in September 1992 with a faecal discharge from the umbilicus. Two years previously, an asymptomatic abdominal mass palpable below the umbilicus had been identified on routine physical examination by his general practitioner. Computerized tomography (CT) studies of the abdomen had revealed that the mass arose from the pelvis, lying caudal to the umbilicus and anterior to the sigmoid colon. Although this structure was filled with air, open communication with the lumen of the sigmoid colon could not be demonstrated on CT scan. A

Correspondence: Stephen G. Pollard, F.R.C.S.

Accepted: 11 January 1993 provisional diagnosis of a giant colonic diver ticulum was made and a conservative approach $\mathbb{\complement}$ adopted since the patient was asymptomatic and in $\vec{B}$ poor general health secondary to diabetes and a3 previous cerebral infarct. A CT scan some 18 months later revealed an air-filled sinus that now communicated with the umbilicus, yet the patiento remained asymptomatic (Figure 1). Five to 6 weeks 3 . prior to admission, the patient experienced constipation with intermittent diarrhoea. This coin $\frac{3}{6}$ cided with the onset of a faecal discharge from hiso umbilicus.

On examination he was afebrile and welle nourished. The abdomen was soft, non-tender, and with a fixed palpable mass in the hypogastrium and? left iliac fossa. Haematological and biochemistryn studies were within normal limits. A barium enema study demonstrated a $7 \mathrm{~cm}$ stricture of the sigmoido colon with a small perforation emptying into the cavity, identified previously by CT scan (Figure 2)

Laparotomy was performed through a midlineo incision and a large cavity in the preperitoneato space was entered. This was not a colonic diver - ? ticulum and was lined with granulation tissue. It communicated with the umbilicus and a large perforation in the sigmoid colon. A sigmoid colec- 